\title{
Self- Assembly of Saponite Nanoparticles Influenced by Interlayer $\mathrm{H}_{2} \mathrm{O}$ Molecules
}

\author{
K. Numata and K. Sato
}

\begin{abstract}
The rheological mechanism of long-term self-assembly caused by $\mathrm{H}_{2} \mathrm{O}$ molecules is highlighted for layered saponite nanoparticles based on the results of positronium (Ps) annihilation spectroscopy and thermogravimetry and differential thermal analysis (TG-DTA). A-type zeolite powder of typical cage material is also investigated to compare with the layered material. Prior to self-assembly, saponite nanoparticles exhibit two kinds of local molecular structures, where one and two nanosheets are inserted into interlayer spaces forming open spaces with their sizes of $\sim 3 \AA$ and $\sim 9 \AA$, respectively. The angstrom-scale open spaces for the saponite vary in the time scale of $\sim 100 \mathrm{~h}$ much longer than that of TG-DTA of $\sim 8 \mathrm{~h}$. The long-term molecular dynamics probed by Ps annihilation spectroscopy originates from the self-assembly of saponite nanoparticles. It is found that the self-assembly of layered saponite nanoparticles is accelerated with increasing the humidity from $35 \%$ to $70 \%$ owing to the formation of two-layer hydration. The present results demonstrate that the long-term self assembly originates from the layered structures together with $\mathrm{H}_{2} \mathrm{O}$ molecules on the layer surfaces.
\end{abstract}

Index Terms-Clay mineral, positronium, nanosheet, local molecular structure, open spaces.

\section{INTRODUCTION}

Nanoparticle self-assembly is a phenomenon, in which nanoscale particles spontaneously agglomerate with well-defined local structures through their mutual interactions [1]. The self-assembly of nanoparticles is increasingly of importance for dealing with the global environmental issues. It has been reported that dynamical functionality in the soil environment emerges as a consequence of self-assembly in the soil-microbe system [2]. In the recent practical model of giant earthquake nucleation, pore fluid of self-assembled geomaterials plays an important role in earthquake slip weakening in plate-boundary faults [3]. In addition, self-assembly often leads to specific properties and functionalities that cannot be accessible by the conventional technique of material synthesis [4]. The application of self-assembly to material synthesis could be thus currently one of the most straightforward strategies that allow the design of new multifunctional nanocomposites [5-6], though the phenomenon of self-assembly itself has not been understood yet.

Manuscript received June 19, 2013; revised July 29, 2013. This work was partially supported by a Grant-in-Aid of the Japanese Ministry of Education, Science, Sports and Culture (Grant Nos. 25400318 and 25400319).

The authors are with Department of Environmental Sciences, Tokyo Gakugei University, 4-1-1 Koganei, Tokyo 184-8501, Japan (e-mail: m121817m@st.u-gakugei.ac.jp; sato-k@u-gakugei.ac.jp).
Inorganic layered compounds known as clay minerals are typical materials that exhibit self-assembly in the presence of $\mathrm{H}_{2} \mathrm{O}$ molecules [7]-[8]. They can contain a large amount of $\mathrm{H}_{2} \mathrm{O}$ molecules in the angstrom-scale interlayer spaces, which is characteristic for the swelling property [9]. The high capability of $\mathrm{H}_{2} \mathrm{O}$ adsorption is based on the fact that the inorganic layered compound possesses a negative layer charge compensated by interlayer cations attractive to polarized $\mathrm{H}_{2} \mathrm{O}$ molecules. Numerous studies on self-assembly of clay nanoparticles have thus been focused on the rheological behavior in aqueous suspension [10]. Layer-by-layer assembly is one of the promising techniques for the fabrication of functional film with respect to the rheology of clay nanoparticles [11], [12]. Synchrotron-based small-angle X-ray scattering for clay nanoparticles in aqueous suspension revealed the strong positional and orientational order of nanosheets, which captures the specific gel structure due to attractive interactions among them [13].

In the present study, the self-assembly of layered-saponite nanoparticles is explored by positronium (Ps) annihilation spectroscopy coupled with thermogravimetry and differential thermal analysis (TG-DTA). We focus on the long-term self-assembly ranging from the onset of $\mathrm{H}_{2} \mathrm{O}$ adsorption at the interlayer cations to far after complete hydration. The purpose of the present work is to answer the following questions: 1. What is the driving force of self-assembly for saponite nanoparticles? 2. Is the self-assembly of saponite nanoparticles rheological? 3. Are there local molecular structures responsible for the self-assembly of saponite nanoparticles?

\section{EXPERIMENTS}

Synthetic Na-type saponite nanoparticles $\left(54.71 \% \quad \mathrm{SO}_{2}\right.$, $5.02 \% \quad \mathrm{Al}_{2} \mathrm{O}_{3}, 0.03 \% \quad \mathrm{Fe}_{2} \mathrm{O}_{3}, 30.74 \% \mathrm{MgO}, 2.15 \% \quad \mathrm{Na}_{2} \mathrm{O}$, $0.07 \% \mathrm{CaO}, 0.67 \% \mathrm{SO} 3,6.64 \% \mathrm{H}_{2} \mathrm{O}$ ) produced by Kunimine Industries Co. Ltd., Japan were employed in this study. The particle size is approximately $45 \mathrm{~nm}$ in diameter. A-type zeolite powder produced by Tosoh Co. Ltd., Japan was employed as well. All the samples were dehydrated at $423 \mathrm{~K}$ for $12 \mathrm{~h}$ under the vacuum condition of $\sim 10^{-5}$ Torr, which are referred as the starting saponite samples.

Adsorption of $\mathrm{H}_{2} \mathrm{O}$ molecules was investigated with respect to the thermogravity (TG) caused by weight gain. The time-dependent TG data were obtained by TG-DTA system (TG-DTA 2020SA, BRUKER AXS Co. Ltd.) at room temperature with $\alpha$ corundum $(\alpha \mathrm{Al} 2 \mathrm{O} 3)$ as an internal standard. The starting samples were exposed to the humidity of $\sim 35 \%$ at the temperature of $\sim 300 \mathrm{~K}$, where the time-dependent data were obtained. 
The sizes of open nanospaces and their fractions were investigated by Ps annihilation lifetime spectroscopy. A fraction of energetic positrons injected into samples forms the bound state with an electron, Ps. Singlet para-Ps ( $p$-Ps) with the spins of the positron and electron antiparallel and triplet ortho-Ps (o-Ps) with parallel spins are formed at a ratio of $1: 3$. Hence, three states of positrons: $p$-Ps, $o$-Ps, and free positrons exist in samples. The annihilation of p-Ps results in the emission of two $\gamma$-ray photons of $511 \mathrm{keV}$ with lifetime 125 ps. Free positrons are trapped by negatively charged parts, such as polar elements, and annihilated into two photons with lifetime $\sim 450$ ps. The positron in $o$-Ps undergoes two-photon annihilation with one of the electrons bound to surrounding molecules with a lifetime of a few ns after localization in angstrom-scale pores. The last process is known as $o$-Ps pick-off annihilation and provides information on the open nanospace size $R$ through its lifetime $\tau_{o-\mathrm{Ps}}$ based on the Tao-Eldrup model [14], [15]:

$$
\tau_{o-P s}=0.5\left[1-\frac{R}{R_{0}}+\frac{1}{2 \pi} \sin \left(\frac{2 \pi R}{R_{0}}\right)\right]^{-1}
$$

where $R_{0}=R+\Delta R$, and $\Delta R=0.166 \mathrm{~nm}$ is the thickness of homogeneous electron layer in which the positron in $o$-Ps annihilates. The positron source $\left({ }^{22} \mathrm{Na}\right)$, sealed in a thin foil of Kapton, was mounted in a sample-source-sample sandwich. The starting samples (dehydrated samples) were exposed to the relative humidity of $\sim 35 \%$ and $\sim 70 \%$ at ambient temperature, where Ps lifetime measurements were performed every 45 min during hydration. Positron lifetime spectra were numerically analyzed using the POSITRONFIT code [16].

\section{RESULTS AND DISCUSSION}

Fig. 1 shows thermogravity (TG) as a function of exposure time. The TG data for saponite gradually increases with increasing exposure time up to $\sim 8 \mathrm{~h}$ owing to hydration in the interlayer spaces and is saturated thereafter. The TG data for zeolite increases more rapidly with exposure time up to $2.5 \mathrm{~h}$ and stays constant thereafter. This demonstrates that $\mathrm{H}_{2} \mathrm{O}$ molecules adsorb more easily for zeolite than saponite.

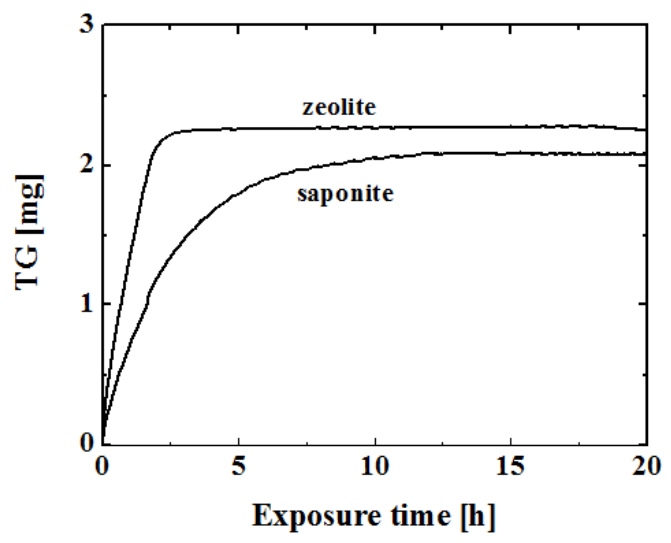

Fig. 1. Thermogravity (TG) data of saponite and zeolite as a function of exposure time.

Ps annihilation spectroscopy for both the saponite and zeolite reveals two kinds open spaces denoted as A and B.
The size of small open space A for saponite is consistently $3 \AA$ without any significant change along with exposure time, whereas the size of large open space B significantly decreases from $\sim 9 \AA$ to $\sim 6 \AA$. For the zeolite sample, small and large open spaces with their sizes of $\sim 3 \AA$ and $\sim 5 \AA$ corresponding to $\beta$ and $\alpha$ cages, respectively, are obtained.

In Fig. 2, the fractions of small and large open spaces obtained for the saponite at the relative humidity of $\sim 35 \%$ and $\sim 70 \%$ are presented together with those of zeolite at $35 \%$ as a function of exposure time. The fraction of small open space for the zeolite quickly increases from $\sim 7 \%$ to $13 \%$ with increasing exposure time along with the decrease of large open space from $\sim 12 \%$ to $\sim 0 \%$. The large open space is not obtained anymore after the exposure time of $2.5 \mathrm{~h}$, which is well synchronized with TG data (see Fig. 1). This is typical behavior that Ps annihilation occurs in water filled $\alpha$ and $\beta$ cages [17]. The present results of Ps annihilation spectroscopy together with TG-DTA clearly capture the picture that the weight increase due to hydration observed for the zeolite solely arises from the occupation of $\alpha$ and $\beta$ cages by $\mathrm{H}_{2} \mathrm{O}$ molecules.

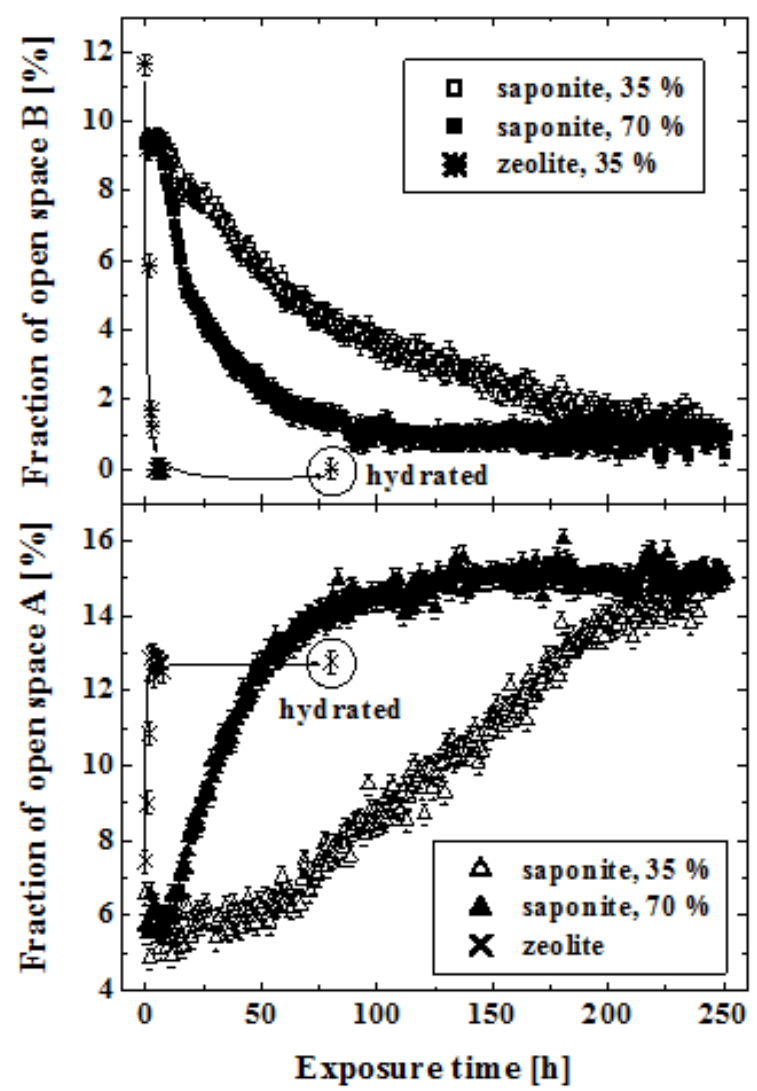

Fig. 2. Fractions of open spaces A and B obtained for saponite obtained at the relative humidity of $\sim 35 \%$ and $\sim 70 \%$ together with those of zeolite as a function of exposure time. The data of well hydrated zeolite are added at the exposure time of $\sim 80 \mathrm{~h}$.

In sharp contrast with the zeolite, the fraction of small open space for the saponite slowly increases from $\sim 5 \%$ to $\sim$ $9 \%$ with exposure time together with the decrease of large open space from $\sim 10 \%$ to $\sim 4 \%$. They are well synchronized in the time scale with $\sim 100 \mathrm{~h}$ much longer than that of TG-DTA with $\sim 8 \mathrm{~h}$. It is thus concluded that the long-term molecular dynamics probed by Ps annihilation spectroscopy originates from the self-assembly of saponite nanoparticles 
rheologically caused by $\mathrm{H}_{2} \mathrm{O}$ molecules. The present finding demonstrates that the long-term self assembly is caused by not the cage but the nanolayered structures.

Our next interest is where the two kinds of open spaces are located in the present saponite nanoparticles under study. The interlayer spaces for the dehydrated saponite completely shrink $\mathrm{Na}^{+}$cations infilling up the hexagonal cavity of silicate tetrahedron, as confirmed by XRD experiments [18-19]. Generally, they expand together with $\mathrm{H}_{2} \mathrm{O}$ molecules adsorbed at the $\mathrm{Na}^{+}$cations in the interlayer spaces in result of hydration. It is thus unlikely that the open space A with the constant size of $\sim 3 \AA$ corresponds to the interlayer spaces. The open space B with its size ranging from $\sim 6$ to $\sim 9 \AA$ is in turn too large to relate with the interlayer spaces. We thus introduced two kinds of local molecular structures, as illustrated in Fig. 2, to explain the long-term variation of open nanospaces observed for the layered saponite. These structures were previously simulated by molecular dynamics (MD) calculations [20]. One of the molecular structures is called type $\mathrm{A}$, in which a one-clay nanosheet is inserted into the interlayer spaces forming the open space with the size of $\sim 0.3 \mathrm{~nm}$. Another is type $\mathrm{B}$, in which a two-clay nanosheet is inserted into the interlayer spaces forming the large open space with the size of $\sim 0.9 \mathrm{~nm}$. Note that the sizes of open spaces simulated by MD calculations are in agreement with those observed by Ps annihilation spectroscopy.

The local molecular structure denoted as the type A with the smaller open space increases maintaining its size along with self-assembly. On the other hand, the local structure of the type B disappears with self-assembly shrinking its larger open space. The type A is thus an intrinsic local molecular structure of saponite nanoparticles, whereas the type B is metastable structure present dominantly prior to self-assembly. It is reasonably inferred that the metastable structure of type B is gradually altered to the intrinsic structure of type A together with self-assembly.

Based on the present findings of Ps annihilation spectroscopy together with TG-DTA, the following rheological mechanism of self-assembly induced by $\mathrm{H}_{2} \mathrm{O}$ molecules can be drawn for saponite nanoparticles. Prior to self-assembly saponite nanoparticles possess two kinds of local molecular structures, where one and two nanosheets are inserted into the interlayer spaces forming the open spaces with their sizes of $\sim 0.3$ and $\sim 0.9 \mathrm{~nm}$, respectively. The local structures with smaller and larger open spaces correspond to the intrinsic and metastable molecular environments, respectively. $\mathrm{H}_{2} \mathrm{O}$ molecules significantly trigger off the self-assembly of saponite nanoparticles. $\mathrm{H}_{2} \mathrm{O}$ molecules adsorbed at the $\mathrm{Na}^{+}$cations in the interlayer spaces act as a lubricant, causing the driving force for the rheological motion of nanosheets in parallel to the layer direction. One of two nanosheets inserted into the interlayer spaces are thus released away (see Fig. 3). The local molecular structures with the larger open spaces are gradually altered to those with the smaller open spaces that finally get to dominant for the self-assembled saponite nanoparticles. The self-assembly of layered saponite nanoparticles is accelerated with increasing the relative humidity from $\sim 35 \%$ to $\sim 70 \%$ owing to two-layer hydration.

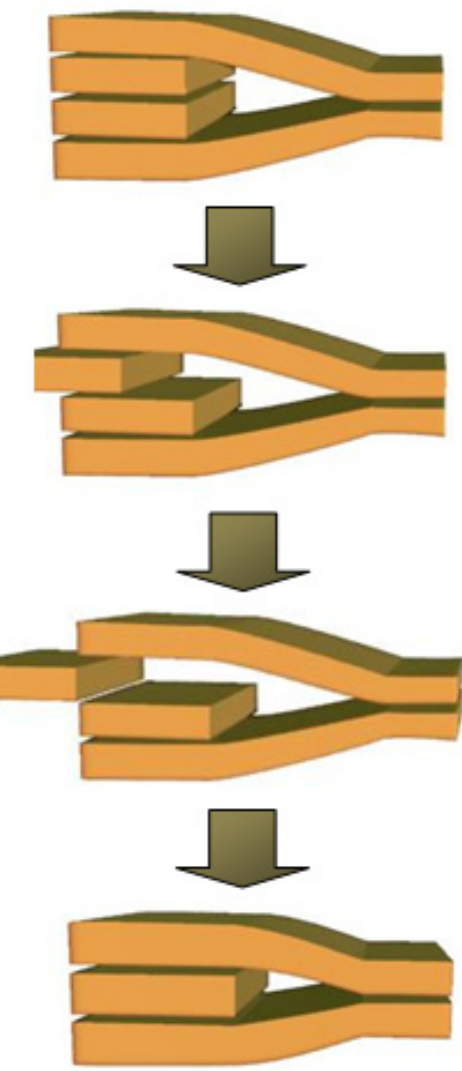

Fig. 3. Schematic illustration of self-assembly caused by two kinds of local molecular structures.
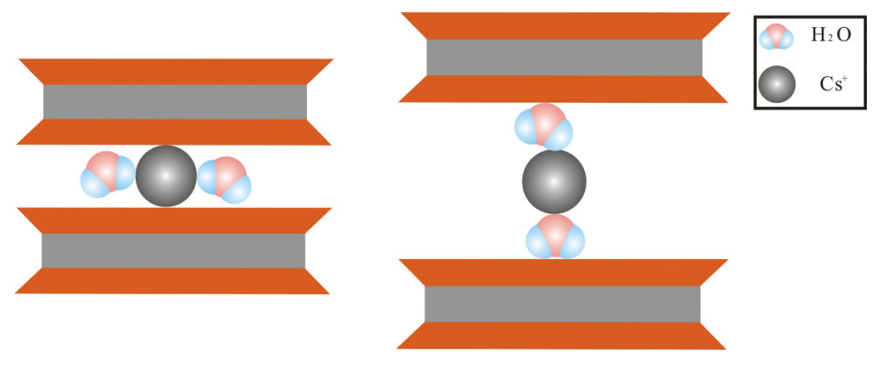

Fig. 4. Schematic illustration of one- and two- $\mathrm{H}_{2} \mathrm{O}$ layer hydration.

It is noted here that the fraction of small open space A observed at the relative humidity of $\sim 70 \%$ increases more rapidly than those at $\sim 35 \%$ with increasing exposure time. The fraction of large open space B decreases at $~ 75 \%$ with exposure time, which is well synchronized in the time scale with $\sim 100$ hours. This clearly demonstrates that the self-assembly is significantly accelerated with increasing the amount of $\mathrm{H}_{2} \mathrm{O}$ molecules due to hydration. According to the recent X-ray diffraction studies on swelling behavior of montmorillonite clay mineral, one $\mathrm{H}_{2} \mathrm{O}$ layer is stacked on the layer surfaces at the relative humidity as $\sim 40 \%$, whereas two $\mathrm{H}_{2} \mathrm{O}$ layers are formed there at the higher relative humidity as $\sim 70 \%$. We reasonably infer that the two $\mathrm{H}_{2} \mathrm{O}$ layers at the surfaces act as lubricant enhancing the self-assembly of layered nanosheet.

\section{CONCLUSION}

The rheological mechanism of long-term self-assembly induced by $\mathrm{H}_{2} \mathrm{O}$ molecules was investigated for 
layered-saponite nanoparticles comparing with A-type zeolite as one of the cage materials. Prior to self-assembly, saponite nanoparticles exhibit two kinds of local molecular structures, where one and two nanosheets are inserted into interlayer spaces forming open spaces with their sizes of $\sim 3$ $\AA$ and $\sim 9 \AA$, respectively. Meanwhile, open spaces with their sizes of $\sim 3 \AA$ and $\sim 5 \AA$ corresponding to $\beta$ and $\alpha$ cages are obtained for the zeolite. The occupation of both $\alpha$ and $\beta$ cages by $\mathrm{H}_{2} \mathrm{O}$ molecules proceeds along with hydration up to $2.5 \mathrm{~h}$, which well synchronizes with the weight gain in TG data. On the contrary, the angstrom-scale open spaces for the saponite vary with hydration in the time scale with $\sim 100 \mathrm{~h}$ much longer than that of TG-DTA with $\sim 8 \mathrm{~h}$. The long-term molecular dynamics probed by Ps annihilation spectroscopy originates from the self-assembly of saponite nanoparticles rheologically caused by $\mathrm{H}_{2} \mathrm{O}$ molecules as follows. $\mathrm{H}_{2} \mathrm{O}$ molecules adsorbed at $\mathrm{Na}^{+}$cations in the interlayer spaces immediately trigger off the onset of the rheological motion of nanosheets in parallel to the layer direction. One of two nanosheets inserted into the interlayer spaces are thus gradually released away, the local molecular structure with smaller open spaces getting to dominant for the self-assembled saponite nanoparticles. It is found that the self-assembly of layered saponite nanoparticles is accelerated with increasing the relative humidity from $\sim 35 \%$ to $\sim 70 \%$ owing to the formation of two-layer hydration. The present results demonstrate that the long-term self-assembly originates from the layered structures together with $\mathrm{H}_{2} \mathrm{O}$ molecules on the layer surfaces. It is beneficial to detect the molecular environment containing the edge site of clay nanosheet specifically in the present study. This site has not been identified by other techniques as e.g., transmission electron microscopy (TEM) and XRD, thus having been unconsidered for the molecular model of inorganic layered nanoparticles so far. It is expected that the edge site is chemically active as that of cup-stacked carbon nanotube. The present findings are therefore of particular importance for further experimental approaches with respect to multifunctional catalytic and adsorption materials in addition to the proper treatment of global environmental issues.

\section{ACKNOWLEDGMENT}

The authors are indebted to K. Fujimoto (Tokyo Gakugei University), M. Nakata (Tokyo Gakugei University), N. Shikazono (Keio Univeristy), K. Kawamura (Okayama University), Y. Kobayashi (National Institute of Advanced Industrial Science and Technology), K. Ito (National Institute of Advanced Industrial Science and Technology), and M. Kamaya (Kogakuin University) for fruitful discussion.

\section{REFERENCES}

[1] G. M. Whitesides and B. Grzybowski, "Self-Assembly at All Scales," Science, vol. 295, pp. 2418-2421, 2002.

[2] I. M. Young and J. W. Crawford, "Interactions and Self-Organization in the Soil-Microbe Complex," Science, vol. 304, pp. 1634-1637, 2004.

[3] C. A. J. Wibberley, T. Shimamoto, "Earthquake slip weakening and asperities explained by thermal pressurization," Nature, vol. 436, pp. 689-692, 2005.

[4] C. W. Chiu and J. J Lin, "Self-assembly behavior of polymer-assisted clays," Progress in Polymer Science, vol. 37, pp. 406-444, 2012.

[5] I. Y. Jeon and J. B. Baek, "Nanocomposites Derived from Polymers and Inorganic Nanoparticles," Materials, vol. 3, pp. 3654-3674, 2010.

[6] I. Vicente, P. Salagre, and Y. Cesteros, "Ni nanoparticles supported on microwave-synthesised saponite for the hydrogenation of styrene oxide," Applied Clay Science, vol. 53, pp. 212-219, 2011.

[7] E. Balnois, S. Durand-Vidal, and P. Levitz, "Probing the morphology of Laponite clay colloids by atomic force microscopy," Langmuir, vol. 19, pp. 6633-6637, 2003.

[8] B. V. Lotsch and G. A. Ozin, "Photonic Clays: A New Family of Functional 1D Photonic Crystals," ACS Nano, vol. 2, pp. 2065-2074, 2008.

[9] P. Porion, L. J. Michot, A. M. Faugére, A. Delville, "Structural and Dynamical Properties of the Water Molecules Confined in Dense Clay Sediments: a Study Combining 2H NMR Spectroscopy and Multiscale Numerical Modeling," J. Phys. Chem. C, vol. 111, pp. 5441-5453, 2007.

[10] R. Bandyopadhyay, D. Liang, H. Yardimci D. A. Sessoms, M. A. Borthwick, S. G. J. Mochrie, J. L. Harden, and R. L. Leheny, "Evolution of Particle-Scale Dynamics in an Aging Clay Suspension," Phys. Rev. Lett., vol. 93, pp. 2283021-2283024, 2004.

[11] G. Decher, "Fuzzy Nanoassemblies: Toward Layered Polymeric Multicomposites," Science, vol. 277, pp. 1232-1237, 1997.

[12] K. Glinel, A. Laschewsky, and A. M. Jonas, "Ordered Polyelectrolyte "Multilayers". 4. Internal Structure of Clay-Based Multilayers," J. Phys. Chem. B, 2002, 106, 11246-11252.

[13] L. J. Michot, I. Bihannic, S. Maddi, S. Funari, C. Baravian, P. Levitz, and P. Davidson, "Liquid Crystalline Aqueous Clay Suspensions," PNAS, vol. 103, pp. 16101-16104, 2006.

[14] S. J. Tao, "Positronium annihilation in molecular substances," J. Chem. Phys., vol. 56, pp. 5499-5510, 1972.

[15] M. Eldrup, D. Lightbody, and J. N. Sherwood, "The temperature dependence of positron lifetimes in solid pivalic acid," Chem. Phys., vol. 63, pp. 51-58, 1981.

[16] P. Kirkegaard and M. Eldrup, "Positronfit extended: A new version of a program for analysing position lifetime spectra," Comput. Phys. Commun., vol. 7, pp. 401-409, 1974.

[17] A. M. Habrowska and E. S. Popiel, "Positron annihilation in zeolite 13X, " J. Appl. Phys., vol. 62, pp. 2419, 1987.

[18] S. Morodome and K. Kawamura, "Swelling behavior of Na- and Ca-montmorillonite up to $150 \mathrm{C}$ by in situ $\mathrm{x}$-ray diffraction experiments," Clay Clay Miner., vol. 57, pp. 150-160, 2009.

[19] S. Morodome and K. Kawamura, "In situ X-ray diffraction study of the swelling of montmorillonite as affected by exchangeable cations and temperature," Clay Clay Miner., vol. 59, pp. 167-175, 2011.

[20] K. Sato, K. Fujimoto, K. Kawamura, W. Dai, and M. Hunger, "Rheological Mechanism of Long-Term Self-Assembly in Saponite Nanoparticles," J. Phys. Chem. C, vol. 116, pp. 22954-22959, 2012.

Kazuomi Numta received his Bachelor degree from Department of Physics, Tokyo University of Science, Japan, in 2012, K. Numata got into the master course of Department of Environmental Sciences, Tokyo Gakugei University. He has concentrated on researches on complex phenomena originated from angstrom- and nano-scale open spaces in material as well as in geological environment. 